\title{
Ressources Alimentaires De Eidolon helvum (Kerr, 1792), Espece Proche De La Menace (Abidjan, Côte d'Ivoire)
}

\author{
Niamien Coffi Jean Magloire \\ Département de Biologie Animale, UFR Sciences Biologiques, \\ Université Peleforo Gon Coulibaly de Korhogo, Korhogo, Côte d’Ivoire \\ Kadjo Blaise \\ Laboratoire de Zoologie et Biologie Animale, UFR Biosciences, \\ Université Félix Houphouët-Boigny, Abidjan, Côte d’Ivoire \\ Koné Kéassemon Cédessia Hervé \\ Département de Biologie Végétale, UFR Sciences Biologiques, \\ Université Peleforo Gon Coulibaly de Korhogo, Korhogo, Côte d'Ivoire \\ N'Goran Kouakou Eliézer \\ Laboratoire de Zoologie et Biologie Animale, UFR Biosciences, \\ Université Félix Houphouët-Boigny, Abidjan, Côte d’Ivoire
}

doi: 10.19044/esj.2016.v13n3p182 URL:http://dx.doi.org/10.19044/esj.2016.v13n3p182

\begin{abstract}
Food resources of the straw-colored fruit bats Eidolon helvum (Kerr, 1792), a near-threaned species in Abidjan-Plateau were studied from August 2007 to July 2008. Faeces collection under sleeping trees and direct observations were made in order to access qualitative composition and temporal distribution of food resources of Eidolon helvum. Results show that 16 species of plants grouped into eight families and classified in three categories of plant organs were exploited by these bats. Among them, three species of plants appeared for the first time in the diet of the frugivorous bats in Côte d'Ivoire. Fruit food resources were most abundant (83\%) and fruits of Ficus genus were dominant (45\%). The distribution of food resources varied with the season.
\end{abstract}

Keywords: Straw-colored fruit bats, diet, season effect, urban area

\section{Résumé}

Les ressources alimentaires des chauves-souris paillées Eidolon helvum (Kerr, 1792), espèce proche de la menace d'Abidjan-Plateau ont été étudiées d'août 2007 à juillet 2008. Des collectes de fèces sous les dortoirs 
journaliers et des observations directes ont été réalisées en vue de connaître la composition qualitative et la distribution temporelle des ressources alimentaires de Eidolon helvum. Les résultats montrent que 16 espèces de plantes regroupées en huit familles et classées en trois catégories d'organes végétaux sont exploitées par ces frugivores. Parmi elles, trois six espèces de plantes sont apparues pour la première fois dans le régime alimentaire des chauves-souris frugivores de Côte d'Ivoire. Les ressources alimentaires fruitières ont été les plus abondantes (83\%). Les fruits du genre Ficus sont dominants (45 \%). La distribution des ressources alimentaires a variée avec la saison.

Mots Clés : Chauves-souris paillées, régime alimentaire, effet de la saison, Milieu urbain.

\section{Introduction}

L'ordre des Chiroptères présente des intérêts d'ordre systématique (Fenton et Simmons, 2015 ; Herkt et al., 2016), écologique (Jones et al., 2009 ; Kunz et al., 2011 ; Meyer et al., 2015), sanitaire (Hayman et al., 2012 ; Schaer et al., 2013 ; Moratelli et Calisher, 2015 ; Plowright et al., 2015; Saéz et al., 2015), économique (Kunz et al., 2011 ; Ghanem et Voigt, 2012 ; Niamien et al., 2015) et de conservation (Kingston, 2013 ; Niamien et al., 2015 ; Mildenstein et al., 2016). Malgré ces multiples intérêts, peu de données écologiques et biologiques concernant ces animaux sont disponibles en raison de leurs activités nocturnes (Kunz et Parson, 2009 ; Herkt et al., 2016).

Les travaux portant sur le régime alimentaire des chauves-souris revêtent un triple intérêt : scientifique, écologique et économique. En effet, les données recueillies renseignent à la fois sur les ressources alimentaires et le milieu (Thomas, 1982, 1988 ; Ritcher et Cumming, 2006 ; Niamien et al., 2009). Ces études permettent également d'apprécier les services écosystémiques joués par ces animaux dans le fonctionnement des écosystèmes (Kunz et al., 2011 ; Meyer et al., 2015 ; Moratelli et Calisher, 2015). En outre, le comportement migratoire et la taille des populations de chauves-souris frugivores, sont liés à la distribution spatio-temporelle des ressources alimentaires (Klingbeil et Willing, 2010 ; Cisneros et al., 2015). Les chauves-souris frugivores consomment plusieurs espèces de plantes dont certaines sont d'importance économique (Kunz et al., 2011; Ghanem et Voigt, 2012 ; Mass et al., 2013 ; Herkt et al., 2016 ; Williams-Guillén et al., 2016). Cependant, leur spectre de ressources alimentaires n'est pas clairement établi (Thomas, 1982, 1988 ; Stier, 2003 ; Niamien et al., 2009). Pour pallier ce déficit d'informations, les travaux sur leur régime alimentaire devraient être explorés en vue de définir une politique de gestion durable. 
En Côte d'Ivoire, l'écologie alimentaire des chauves-souris frugivores est peu documentée. En effet, seuls quelques rares travaux portant sur le régime alimentaire ont été effectués à Lamto (Thomas, 1982) et à Abidjan (Niamien et al., 2009). Ainsi, aucune information sur les ressources alimentaires des chauves-souris paillées, Eidolon helvum (Kerr, 1792), espèce proche de la menace de la Commune Plateau à Abidjan n'est disponible.

La présente étude, est une contribution à une meilleure connaissance de l'écologie des chauves-souris frugivores afin d'en assurer une gestion rationnelle et durable. Elle vise spécifiquement à analyser la nature des ressources alimentaires exploitées par les chauves-souris paillées Eidolon helvum, espèce proche de la menace et à examiner l'influence des saisons sur leur distribution afin d'en dégager des implications pour sa conservation.

\section{Matériel et méthodes} Site d'étude

La Commune du Plateau s'étend entre les longitudes Ouest $4^{\circ} 10^{\prime}$ et $4^{\circ} 50^{\prime}$ et les latitudes Nord $5^{\circ} 10^{\prime}$ et $5^{\circ} 80^{\prime}$, sur une superficie de $2,5 \mathrm{~km}^{2}$. Elle est située au centre de la ville d'Abidjan, elle-même localisée dans le sud de la Côte d'Ivoire (Figure 1). Dans sa partie centrale, les avenues sont bordées d'arbres d'espèces différentes, qui sont fréquemment utilisés comme dortoirs par la population de chauves-souris paillées (Figure 1). L’intense animation de cette cité administrative les jours ouvrables, contraste fortement avec son calme des jours fériés.

La ville d'Abidjan appartient à une zone caractérisée par un climat à quatre saisons : une petite saison sèche (août à septembre), une petite saison des pluies (octobre à novembre), une grande saison sèche (décembre à mars) et une grande saison des pluies (avril à juillet) (Brou, 1997 ; Niamien, 2011).

\section{Approches méthodologiques Collecte des fèces}

Les ressources alimentaires des chauves-souris paillées de la Commune du Plateau à Abidjan ont été étudiées en analysant la composition de leurs fèces $(\mathrm{N}=12$ 016). Les matières fécales ont été collectées d'août 2007 à juillet 2008 sous les sites dortoirs journaliers (Figure 1). Les excréments ont été collectés à l'aide d'une spatule et mis dans des piluliers référencés portant la date et le site. Les collectes ont eu lieu préférentiellement les dimanches pour tenir compte des activités de balayage des trottoirs de la Commune du Plateau, qui s'y déroulent les jours ouvrables, susceptibles d'éliminer les selles excrétées par les chauves-souris. Par ailleurs, ces données ont été complétées par des observations directes ponctuelles des chauves-souris paillées se nourrissant sur des arbres au sein 
de la zone d'étude. Cette approche a permis de dresser qualitativement la liste des items exploités (Ritcher et Cumming, 2006).

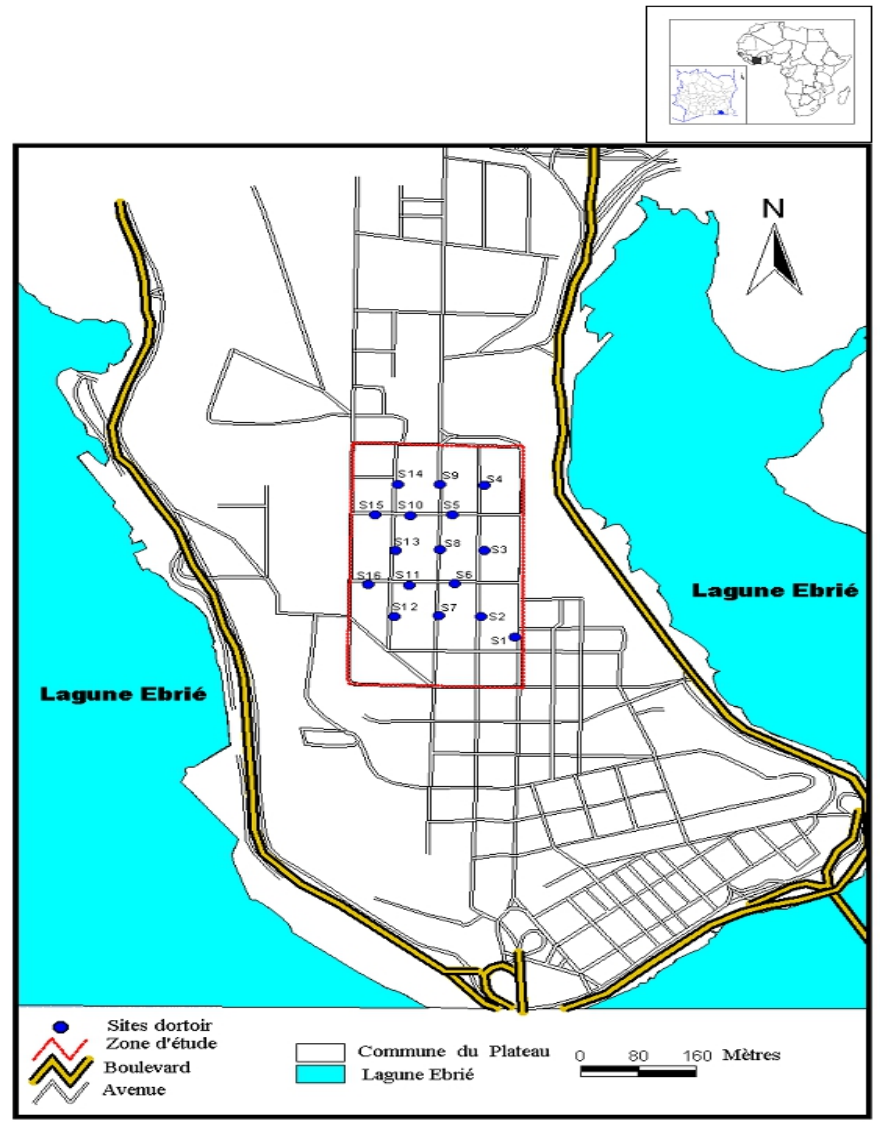

Figure 1 : Cartographie des sites dortoir (numérotés de 1 à 16) de Eidolon helvum dans la commune du Plateau à Abidjan d’août 2007 à juillet 2008.

\section{Tri et identification des ressources alimentaires}

Au laboratoire, le contenu de chaque pilulier a été renversé sur un tamis de $1 \mathrm{~mm}$ de maille, puis lavé avec de l'eau pour isoler les graines, qui ont ensuite été observées et identifiées sous loupe binoculaire. Les identifications ont été faites à l'aide des clés de Hutchinson et Dalziel (1963) et de Aké-Assi $(2001,2002)$ d'une part, et la collection de référence du Centre National de Floristique de l’Université Félix Houphouët-Boigny d'Abidjan d'autre part. Les fèces uniquement constituées de pulpe ont été déterminées sur la base de leur couleur, leur texture et grâce à l'odorat (Thomas, 1982, 1984, 1988). Ces données sont complétées par des observations directes à la jumelle en observant les chauves-souris exploitant des ressources végétales. Dans cette étude, les déterminations se sont faites au niveau de la famille et de l'espèce. 


\section{Expression des résultats}

\section{Pourcentage d'occurrence (F)}

Le pourcentage d'occurrence (F) a été utilisé pour quantifier la fréquence d'apparition des espèces de plantes dans les fèces des chauvessouris paillées. Cette fréquence correspond au pourcentage de relevés de fèces où une espèce de plante est présente par rapport au nombre total de fèces examinés. Son expression est la suivante :

$$
\mathrm{F}=\frac{\mathrm{Si}}{\mathrm{St}} \times 100
$$

Où : $\mathrm{Si}=$ nombre de relevés où l'espèce $\mathrm{i}$ a été présente et $\mathrm{St}=$ nombre total de relevés.

Selon Djakou et Thanon (1988), le pourcentage d'occurrence permet de distinguer cinq classes : les espèces très fréquentes (80 à $100 \%$ ); les espèces fréquentes (60 à $79 \%$ ); les espèces assez fréquentes (40 à $59 \%$ ); les espèces accessoires (20 à $39 \%)$ et les espèces accidentelles $(<20 \%)$.

\section{Indice de diversité de Shannon-Weaver $\left(\mathbf{H}^{\prime}\right)$}

L'indice de diversité de Shannon (H') calculé sur la base de l'abondance relative de chaque espèce végétale présente dans les fèces des chauves-souris paillées (Ramade, 2012), a permis de déterminer le mois et la saison où les ressources alimentaires ont été les plus diversifiées. Cet indice varie de 1 à 5 . Son expression est la suivante :

$\mathrm{H}^{\prime}=-\sum(\mathrm{ni} / \mathrm{N}) \ln (\mathrm{ni} / \mathrm{N})$

$\ln =$ logarithme népérien,

$\mathrm{ni}=$ nombre de fèces mensuel contenant un item $\mathrm{i}$,

$\mathrm{N}=$ nombre total de fèces examinées.

\section{Test statistique}

Avant toute analyse, la distribution normale des données a été vérifiée en utilisant le test de Shapiro-Wilk. En outre, le test de corrélation de Rang de Spearman a été utilisé pour tester le degré de corrélation entre la richesse spécifique et la diversité des ressources alimentaires des chauvessouris paillées. Ces analyses ont été réalisées avec le Logiciel Statistica (version 7.1).

\section{Résultats}

\section{Inventaire des items alimentaires consommés par les chauves-souris paillées}

L’inventaire des items alimentaires trouvés dans les fèces et les observations directes de chauves-souris en fourragement entre août 2007 et juillet 2008 ont permis d'identifier trois catégories d'organes dans le régime 
alimentaire des chauves-souris paillées de la Commune du Plateau à Abidjan : les feuilles, les fleurs et les fruits (Tableau 1). Parmi ces organes végétaux, les fruits sont les plus fréquemment observés (83\%), suivis des feuilles (11\%) et des fleurs (6\%) $(\mathrm{N}=12$ 020; Figure 2).

Tableau 1 : Espèces et organes de plantes consommés par Eidolon helvum dans la Commune du Plateau à Abidjan d'août 2007 à juillet 2008.

\begin{tabular}{ccc}
\hline Familles & Espèces & Organes végétaux \\
\hline ANACARDIACEAE & Mangifera indica $\mathrm{L}$. & Fruit \\
ARAUCARIACEAE & Araucaria excelsa Lamb. & Feuille \\
ARECACEAE & Elaeis guineensis Jacq. & Fruit \\
CARICACEAE & Carica papaya $\mathrm{L}$. & Fruit \\
MIMOSACEAE & Parkia biglobosa Jacq. & Fleur \\
MORACEAE & Ficus benjamina $\mathrm{L}$. & Fruit \\
& Ficus exasperata Vahl & Fruit \\
& Ficus lutea Vahl & Fruit \\
& Ficus polita Vahl & Fruit \\
& Ficus sp. N=1) & Fruit \\
MYRTACEAE & Ficus sur Forssk. & Fruit \\
& Ficus umbellata Vahl & Fruit \\
& Eugenia jambos $\mathrm{L}$. & Fruit \\
POLYPODIACEAE & Eugenia malaccensis $\mathrm{L}$. & Fruit \\
& Psidium guajava $\mathrm{L}$. & Fruit \\
& Platycerium sp. (N=1) & Feuille \\
\hline
\end{tabular}

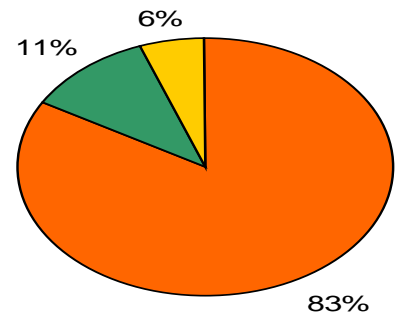

$\square$ Fruits $\square$ Feuilles $\square$ Fleurs

Figure 2 : Proportions des différents organes végétaux consommés par Eidolon helvum dans la commune du Plateau d’août 2007 à juillet 2008 (N=12 020).

Seize espèces de plantes regroupées en huit familles ont été inventoriées. Il s’agit de la famille des Anacardiaceae, des Araucariaceae, des Arecaceae, des Caricaceae, des Mimosaceae, des Moraceae, des Myrtaceae et des Polypodiaceae (Tableau 1). La famille des Moraceae est la plus représentée avec sept espèces de Ficus (45 \%). Elle est suivie de la famille des Myrtaceae avec trois espèces (19\%), les autres familles ne renfermant chacune qu'une seule espèce, soit $6 \%$ chacune (Figure 3 ). Les Ficus sont prédominants dans le régime alimentaire. 


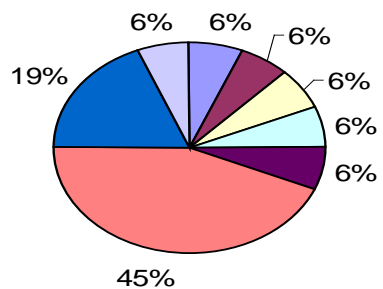

$\begin{array}{lll}\square \text { ANACARDIACEAE } & \square \text { ARAUCARIACEAE } & \square \text { ARECACEAE } \\ \square \text { CARICACEAE } & \square \text { MIMOSACEAE } & \square \text { MORACEAE } \\ \square \text { MYRTACEAE } & \square \text { POLYPODIACEAE } & \end{array}$

Figure 3 : Représentativité des familles végétales dans le régime alimentaire de Eidolon helvum dans la Commune du Plateau à Abidjan d'août 2007 à juillet 2008 (N=12 020).

\section{Pourcentage d'occurrence des espèces végétales dans les fèces de Eidolon helvum}

Le classement des ressources alimentaires des chauves-souris paillées (fruit et feuille) en fonction de leur pourcentage d'occurrence dans les fèces a permis d'identifier cinq classes d'espèces végétales. Les espèces végétales Ficus polita (100\%), Ficus lutea (100\%), Ficus exasperata (100\%), Carica papaya (100 \%) et Mangifera indica (83,33 \%) sont très fréquentes. Les espèces de plantes Psidium guajava (75\%) et Ficus sp. (75 \%) sont fréquentes. Les ressources alimentaires Ficus sur (58,33\%) et Araucaria excelsa (41,66 \%) sont assez fréquentes tandis que Ficus benjamina (16,16 \%), Ficus umbellata (16,16 \%) et Elaeis guineensis (16,16 \%) sont des espèces accidentelles (Tableau 2).

\section{Présence-absence des ressources alimentaires dans les fèces de Eidolon helvum}

Les données de présence-absence des ressources alimentaires dans les fèces de chauves-souris paillées (fruits et feuilles) ont permis de déterminer quatre espèces régulières : Carica papaya, Ficus exasperata, Ficus lutea et Ficus polita. Trois espèces sont quasi-régulières: Ficus sp., Mangifera indica et Psidium guajava. Quatre ressources alimentaires ne sont observées que pendant des saisons précises : Ficus sur (petite saison des pluies et grande saison sèche), Araucaria excelsa (petite saison des pluies et grande saison sèche), Ficus benjamina et Ficus umbellata (grande saison sèche et grande saison des pluies). La ressource alimentaire Elaeis guineensis est la seule espèce ponctuelle (Tableau 2).

Variation temporelle de la richesse et de la diversité spécifique des ressources alimentaires 
La variation mensuelle de la richesse spécifique des ressources alimentaires des chauves-souris paillées montre que le nombre d'espèces a été constant d'août à septembre (petite saison sèche) (7 espèces). Elle a augmenté d'octobre à novembre (petite saison des pluies) (de 8 à 9 espèces), s'est stabilisée jusqu'en février (grande saison sèche) avant de baisser progressivement de mars à juillet (grande saison sèche et grande saison des pluies) (de 9 à 6 espèces) (Tableau 2).

L’indice de diversité de Shannon (H') calculé sur la base des abondances relatives des espèces a augmenté d'août (petite saison sèche) $(1,8)$ à février $(2,3)$, mois au cours duquel il a atteint son pic (grande saison sèche). Cet indice a chuté de mars (grande saison sèche) $(2,01)$ à juin (grande saison des pluies), où le minimum est atteint $(1,5)$. Enfin, cette diversité a augmenté en juillet $(1,6)$ (Tableau 2).

Le test de corrélation de Spearman révèle une forte corrélation entre la richesse spécifique des ressources alimentaires et leur diversité spécifique $(\mathrm{r}=0,91 ; \mathrm{p}<0,001)$.

\section{Discussion}

Les inventaires de la nature des ressources alimentaires de Eidolon helvum de la Commune du Plateau à Abidjan, ont permis d'identifier 16 espèces de plantes. Ce nombre est supérieur à ceux obtenus à Lamto sur les Mégachiroptères qui était de 10 (Thomas, 1982), et de Niamien et al. (2009), qui ont identifié 13 espèces de plantes. Cette différence pourrait résulter de la diversité des ressources alimentaires susceptibles d'être exploitées en zone forestière et particulièrement dans la périphérie du district d'Abidjan. Ainsi, trois nouvelles espèces se sont-elles ajoutées à la liste du régime alimentaire de Eidolon helvum. Il s'agit de Eugenia jambos L. (Myrtaceae), de Eugenia malaccensis L. (Myrtaceae) et de Platycerium sp. (Polypodiaceae). Niamien et al. (2009) n’ont pas identifié ces espèces car la seule méthode utilisée était la collecte des fèces sous les dortoirs. Or, ces espèces végétales ont été déterminées lors de l'observation directe des chauves-souris paillées en nutrition dans la Commune du Plateau à Abidjan.

Tableau 2: Estimation de la fréquence d'apparition des organes de 16 espèces de plantes dans le régime alimentaire des chauves-souris paillées de la Commune du Plateau à Abidjan en fonction des données de présence ou d'absence de ces organes dans les fecès entre août 2007 et juillet 2008. (+ : Présence ; - : absence ; PSS : petite saison sèche ; PSP : petite saison des pluies, GSS : grande saison sèche et $\mathbf{G S P}$ : Grande saison des pluies ; $\mathbf{S}$ : nombre d'espèces ; H' : Indice de Shannon-weaver). 


\begin{tabular}{|c|c|c|c|c|c|c|c|c|c|c|c|c|c|c|c|c|c|}
\hline Espèces & Août & Septembre & PSS & Octobre & Novembre & PSP & Décembre & Janvier & Février & Mars & GSS & Avril & Mai & Juin & Juillet & GSP & $\begin{array}{c}\text { Classification en } \\
\text { fonction du } \\
\text { pourcentage }\end{array}$ \\
\hline $\begin{array}{c}\text { Araucaria } \\
\text { excelsa }\end{array}$ & - & - & - & - & + & + & + & + & + & + & + & - & - & - & - & - & $\begin{array}{c}\text { Assez fréquente } \\
(41,66)\end{array}$ \\
\hline Carica papaya & + & + & + & + & + & + & + & + & + & + & + & + & + & + & + & + & $\begin{array}{c}\text { Très fréquente } \\
\text { (100) }\end{array}$ \\
\hline $\begin{array}{c}\text { Elaeis } \\
\text { guineensis }\end{array}$ & - & - & - & - & - & - & - & - & + & + & + & - & - & - & - & - & $\begin{array}{c}\text { Accidentelle } \\
(16,66)\end{array}$ \\
\hline $\begin{array}{c}\text { Ficus } \\
\text { benjamina }\end{array}$ & - & - & - & - & - & - & + & - & - & - & + & - & - & + & - & + & $\begin{array}{c}\text { Accidentelle } \\
(16,66)\end{array}$ \\
\hline $\begin{array}{c}\text { Ficus } \\
\text { exasperata }\end{array}$ & + & + & + & + & + & + & + & + & + & + & + & + & + & + & + & + & $\begin{array}{c}\text { Très fréquente } \\
\text { (100) }\end{array}$ \\
\hline Ficus lutea & + & + & + & + & + & + & + & + & + & + & + & + & + & + & + & + & $\begin{array}{c}\text { Très fréquente } \\
\text { (100) }\end{array}$ \\
\hline Ficus polita & + & + & + & + & + & + & + & + & + & + & + & + & + & + & + & + & $\begin{array}{c}\text { Très fréquente } \\
\text { (100) }\end{array}$ \\
\hline Ficus sp. & + & - & + & + & + & + & + & + & + & + & + & + & + & - & - & + & Fréquente (75) \\
\hline Ficus sur & + & + & + & + & + & + & + & + & + & - & + & - & - & - & - & - & $\begin{array}{c}\text { Assez fréquente } \\
(58,83)\end{array}$ \\
\hline $\begin{array}{c}\text { Ficus } \\
\text { umbellata }\end{array}$ & - & - & - & - & - & - & - & + & - & - & + & + & - & - & - & + & $\begin{array}{c}\text { Accidentelle } \\
(16,66)\end{array}$ \\
\hline $\begin{array}{l}\text { Mangifera } \\
\text { indica }\end{array}$ & - & + & + & + & + & + & - & + & + & + & + & + & + & + & + & + & $\begin{array}{c}\text { Très fréquente } \\
(83,33)\end{array}$ \\
\hline $\begin{array}{l}\text { Psidium } \\
\text { guajava } \\
\end{array}$ & + & + & + & + & + & + & + & - & + & + & + & + & - & - & + & + & Fréquente (75) \\
\hline $\begin{array}{c}\text { Richesse } \\
\text { spécifique (S) }\end{array}$ & 7 & 7 & 8 & 8 & 9 & 9 & 9 & 9 & 10 & 9 & 12 & 8 & 6 & 6 & 6 & 9 & \\
\hline $\begin{array}{c}\text { Diversité } \\
\text { spécifique (H') }\end{array}$ & 1,8 & 1,9 & 1,9 & 2,04 & 2,05 & 2 & 2,15 & 2,1 & 2,3 & 1,8 & 2,3 & 1,7 & 1,6 & 2 & 1,65 & 1,7 & \\
\hline
\end{tabular}


Les différents organes végétaux (feuilles, fleurs et fruits) constituants les ressources alimentaires des chauves-souris paillées de la Commune du Plateau à Abidjan sont de la même nature que ceux observés à Lamto et à Ouango-Fitini (Thomas, 1982, 1988), au Mont Nimba (Wolton et al., 1982) et aux Philippines (Stier, 2003). A l'instar de nos résultats, divers auteurs indiquent également une dominance des ressources fruitières (Thomas, 1982, 1988 ; Wolton et al., 1982 ; Stier, 2003 ; Richter et Cumming, 2006).

La prédominance des espèces du genre Ficus dans le régime alimentaire des chauves-souris frugivores n’est pas spécifique aux chauvessouris paillées (Eidolon helvum) de la Commune du Plateau à Abidjan. En effet, ce constat a été fait sur la communauté de Mégachiroptères de Lamto (Thomas, 1982, 1988) et sur les colonies de Acerodon jubatus et de Pteropus vampyrus aux Philippines (Stier, 2003). Au contraire, les chauves-souris paillées du Parc National de Kasanga en Zambie, ont une consommation marquée pour les genres Uapaca et Syzygium (Richter, 2004). La grande consommation des espèces du genre Ficus serait liée à leur abondance et à leur bonne valeur calorifique. En effet, ces ressources alimentaires sont très abondantes, bien distribuées et facilement repérables, d’où la réduction du temps de recherche et de la dépense énergétique associée ainsi que de la compétition alimentaire (Hagen et Sabo, 2011 ; Cisneros et al., 2015). Par ailleurs, ces fruits riches en protéines couvriraient leurs besoins énergétiques journaliers (Thomas, 1982, 1984, 1988 ; Durant et al., 2013 ; Cisneros et al., 2015).

Les données sur la présence-absence des ressources alimentaires dans les fèces des chauves-souris paillées ont permis d’identifier trois catégories d’espèces végétales, régulières, saisonnières et ponctuelles. Selon certains auteurs, les fruits des espèces du genre Ficus seraient présentes toute l'année dans les zones de fourragement (Thomas, 1982 ; Yamagiwa et al., 1996 ; Siter, 2003 ; Goné bi, 2007). Nos résultats confirment ce fait pour les espèces de Ficus exasperata, de Ficus lutea et de Ficus polita, contrairement aux autres espèces du genre Ficus, qui sont saisonnières. L'exploitation saisonnière de ces espèces pourrait être liée à leur disponibilité et à leur distribution dans des zones particulières. En effet, l'exploitation d'une espèce est liée à sa disponibilité (Ritcher et Cumming, 2006 ; Fahr et Kalko, 2011 ; Cisneros et al., 2015).

Dans le cadre de cette étude, nous avons eu recours aux méthodes d'analyse des fèces de chauves-souris paillées collectées chaque semaine sous les arbres dortoirs d'une part, et à l'observation directe d'individus se nourrissant d'autre part. Bien que ces méthodes présentent un avantage en termes de coût, elles ne sont pas assez fines pour identifier la fraction de fleurs consommée. Ces approches devraient donc être complétées par la capture des individus aux filets afin de rechercher d'éventuelles traces de 
pollen dans la fourrure de ces pollinivores et en identifier les espèces de plantes (Thomas, 1982).

\section{Conclusion}

L'étude des ressources alimentaires des chauves-souris paillées, Eidolon helvum, une espèce proche de la menace de la Commune du Plateau à Abidjan a relevé que 16 espèces de plantes sont consommées. Parmi celles-ci, trois sont nouvelles dans le régime alimentaire de Eidolon helvum. Il s’agit de Eugenia jambos (Myrtaceae), de Eugenia malaccensis (Myrtaceae) et de Platycerium sp. (Polypodiaceae). Trois catégories d’organes végétaux représentées par les fruits, les feuilles et les fleurs sont exploitées par ces Mégachiroptères. Les ressources alimentaires du genre Ficus sont prédominantes. Carica papaya, Ficus exasperata, Ficus lutea et Ficus polita ont été des ressources alimentaires régulières alors que Ficus sp., Mangifera indica et Psidium guajava ont été quasi-régulières. Ficus benjamina et Ficus umbellata ont été des espèces saisonnières. La richesse spécifique et la diversité des ressources alimentaires dans les fèces ont été les plus élevées en grande saison sèche. Pour assurer la protection de cette population de chauves-souris paillées proche de la menace, la localisation des sites de nutrition devrait être envisagée. Par ailleurs, la protection des espèces d'arbre dont les fruits constituent leurs ressources alimentaires doit être envisagée avec une attention particulière pour les espèces régulières dans les fèces (Carica papaya (présente dans la Commune du Plateau à Abidjan), Ficus exasperata, Ficus lutea et Ficus polita), quasi-régulières (Ficus sp., Mangifera indica et Psidium guajava (présente dans la Commune du Plateau à Abidjan)) et saisonnières (Ficus benjamina et Ficus umbellata). Ces mesures devraient permettre de réduire les dégâts causés par ces frugivores sur les vergers environnants et d'assurer le maintien de cette population de Eidolon helvum sur son site naturel de la Commune du Plateau à Abidjan utilisé comme site dortoir et de reproduction mais aussi comme nurseries.

\section{References:}

1. Aké-Assi, L. (2001). Flore de la Côte d'Ivoire, tome 1: Catalogue systématique, biogéographique et écologique. Mémoires de Botanique systématique. Edition Boissiera, 396p.

2. Aké-Assi, L. (2002). Flore de la Côte d’Ivoire, tome 2: Catalogue systématique, biogéographique et écologique. Mémoires de Botanique systématique. Edition Boissiera, 401p.

3. Brou, Y. (1997). Analyse et dynamique de la pluviométrie en milieu forestier Ivoirien. Thèse de troisième cycle. Abidjan-Cocody, Côted’Ivoire, Université d'Abidjan-Cocody. 
4. Cisneros, L. M., Fagan M. E., \& Willig, M. R. (2015). Effects of human-modified landscapes on taxonomic, functional, and phylogenetic dimensions of bat biodiversity. Diversity and Distributions, 21: 523-533.

5. Djakou, R., \& Thanon, S.Y. (1988). Ecologie Afrique intertropicale. Edition Bordas, 191 p.

6. Durant, K. A., Hall, R. W., Cisneros, L. M., Hyland, R. M., \& Willig, M. R. (2013). Reproductive phenologies of phyllostomid bats in Costa Rica. Journal of Mammalogy, 94: 1438-1448.

7. Fahr, J., \& Kalko, E. K. V. (2011). Biome transitions as centres of diversity: habitat heterogeneity and diversity of West African bat assemblage across spatial scales. Ecography, 34: 177-195.

8. Fenton, M. B., \& Simmons, N. B. (2015). Bats, a world of science and mystery. The University of Chicago Press, 303 p.

9. Ghanem, S. H., \& Voigt, C. C. (2012). Increasing awareness of ecosystem services provided by bats. Advances in the Study of Behavior, 44: 279-302.

10. Goné-bi, Z. B.(2007). Régime alimentaire, distribution spatiale et phénologie des plantes dont

11. les fruits sont consommés par les Chimpanzés du Parc national de Taï (Côte d’Ivoire). Thèse de Doctorat d'Ecologie végétale. AbidjanCocody, Côte d'Ivoire, Université d'Abidjan-Cocody.

12. Hagen, E. M., \& Sabo, J. L. (2011). A landscape perspective on bat foraging ecology a-long rivers: does channel confinement and insect availability influence the response of bats to aquatic resources in riverine landscapes? Oecologia, 166: 751-760.

13. Hayman, D.T.S., Yu, M., Crameri, G., Wang, L., Suu-ire, R., Wood, J.L.N., \& Cunningham, A.A. (2012). Ebola virus antibodies in fruit bats, Ghana, West Africa. Journal of Infection Diseases, 18 (7): 1207-1209.

14. Herkt, K. M. B., Barnikel, G. K., Skidmore, A., \& Fahr, J. (2016). A high-resolution model of bat diversity and endemism for continental Africa. Ecological Modelling, 320: 9-28.

15. Hutchinson, J., \& Dalziel, J. M. (1963). Flora of west tropical Africa. Crown Agents for oversea governments and Administration, 544p.

16. Jones, G., Jacobs, D.S., Kunz, T.H., Willig, M.R., \& Racey, P.A. (2009). Carpe noctem: the importance of bats as bioindicators. Endangered Species Research, 8: 93-115.

17. Kingston, T. (2013). Response of bat diversity to forest disturbance in Southeast Asia: insights from long-term research in Malaysia. In R. A. Adams \& S. C. Pedersen (Ed.), Bat evolution, ecology and conservation (pp. 169-185). New York, NY: Springer. 
18. Klingbeil, B.T., \& Willig, M. R. (2010). Seasonal differences in population assemblage and community level responses of bats to landscape structure in Amazonia. Oikos, 119 :1654-1664.

19. Kunz, T.H., Braun de Torrez, E., Bauer, D., Lobova, T., \& Fleming, T.H. (2011). Ecosystem services provided by bats. Annals of the New York Academy of Sciences, 1223:1-38.

20. Kunz, T. H., \& Parsons, S. (2009). Ecological and Behavioural Methods for the study of bats $2^{\text {nd }}$ Edition. The Johns Hopkins University press, 901p.

21. Maas, B., Clough, Y., \& Tscharntke, T. (2013). Bats and birds increase crop yield in tropical agroforesty landscapes. Ecology letters, 16 (12):1480-1487.

22. Meyer, C. F. J., Aguiar, L. M. S., Aguirre, L. F., Baumgarten, J., Clarke, F. M., Cosson, J. F., Villegas, S. E., Fahr, J., Faria, D., Furey, N., Henry, M. L., Jenkins, R. K. B., Kunz, T. H., Mc. Gonzalez, S. M. C., Moya, I., Pons, J. M., Racey, P. A., Rex, K., Sampaio, E. M., Stoner, K. E., Voigt, C. C., Staden, D. V., Weise, C. D., \& Kalko E. K. V. (2015). Species undersampling in tropical bat surveys: Effects on emerging biodiversity patterns. Journal of Animal Ecology, 84: 113-123.

23. Mildenstein, T., Tanshi, I., \& Racey, P. A. (2016). Exploitation of Bats for Bushmeat and Medicine. In C. C. Voigt, \& T. Kingston (Ed.), Bats in the Anthropocene: conservation of bats in a changing world (pp. 325 -375). New York, NY: Springer.

24. Moratelli, R., \& Calisher, H. C. (2015). Bats and zoonotic viruses: Can we confidently link bats with emerging deadly viruses? Memórias do Instituto Oswaldo Cruz, 110 (1): 1-22.

25. Niamien, C. J. M. (2011). Distribution spatio-temporelle, régime alimentaire, dynamique de population et menaces sur les chauvessouris frugivores de la commune du Plateau à Abidjan (Côte d'Ivoire). Thèse de Doctorat d'Ecologie Tropicale. Abidjan-Cocody, Côte d'Ivoire, Université d'Abidjan-Cocody.

26. Niamien, C. J. M., Yaokokoré-Béibro, K. H., Koné, I., Yao, S. S., \& N’Goran, K. E. (2009). Données préliminaires sur les habitudes alimentaires des chauves-souris paillées, Eidolon helvum (Kerr, 1792) (Chiroptera : Pteropodidae) de la commune d'Abidjan-Plateau (Côte d'Ivoire). Agronomie Africaine, 21 (3) : 231-240.

27. Niamien, C. J. M., Kadjo, B., Dago, D. N., Koné, I., \& N’Goran, K. E. (2015). Initial data on poaching of Eidolon helvum (Kerr, 1792) near-threatened species in Côte D'Ivoire, West Africa. European Journal of Scientific Research, 135 (2): 219-227. 
28. Plowright, R. K., Eby, P., Hudson, P. J., Smith, I. L., Westcott, D., Bryden, W. L., Middleton, D., Reid, P. A., Mc Farlane, R. A., Martin, G., Tabor, G. M., Skerratt, L. F., Anderson, D. L., Crameri, G., Quammen, D., Jordan, D., Freeman, P., Wang, L. F., Epstein, J.H., Marsh, G.A., Kung, N.Y., \& Mc Callum H. (2015). Ecological dynamics of emerging bat virus spillover. Biological Sciences, 282: 2014-2124.

29. Ramade, F. (2012). Éléments d’écologie : Ecologie appliquée, 7ème édition. Edition Dunod, 824p.

30. Richter, H.V. (2004). The foraging ecology of fruit bats in the seasonal environment of central Zambia. Master of Sciences. Florida, USA, University of Florida.

31. Richter, H. V., \& Cumming, G. S. (2006). Food availability and annual migration of the straw-colored fruit bat (Eidolon helvum)," Journal of Zoology, 268 (1): 35-44.

32. Saéz, A. M., Weiss, S., Nowak, K., Lapeyre, V., Zimmermann, F., Düx, A., Kühl, H. S., Kaba, M., Regnaut, S., Merkel, K., Sachse, A., Thiesen, U., Villányi, L., Boesch, C., Dabrowski, P.W., Radonić, A., Nitsche, A., Leendertz, S. A. J., Petterson, S., Becker, S., Krähling, V., Couacy-Hymann, E., Akoua-Koffi, C., Weber, N., Schaade, L., Fahr, J., Borchert, M., Gogarten, J. F., Calvignac-Spencer, S., \& Leendertz, F. H. (2015). Investigating the zoonotic origin of the West African Ebola epidemic. Embo Molecular Medicine, 7 (1):17-23.

33. Schaer, J., Perkins, S. L., Decher, J., Leendertz, F. H., Fahr, J., Weber, N., \& Matuschewski, K. (2013). High diversity of West African bat malaria parasites and a tight link with rodent Plasmodium taxa. PNAS, 110 (43) : 17415-17419.

34. Stier, S.C. (2003). Dietary habitats of two threatened co-roosting flying foxes Subic bay, Philippines. Master of Science. Montana, USA, University of Montana.

35. Thomas, D.W. (1982). The Ecology of an African savanna fruit bat community: Resource partitioning and role in seed dispersal. PhD. Aberdeen, Scotland, University of Aberdeen.

36. Thomas, D.W. (1984). Fruit intake and energy budgets of frugivorous bats. Journal of Physiology Zoology, 57 (4): 457-467.

37. Thomas, D.W. (1988). Analysis of diets of plant-visiting bats. In T. H. Kunz (Ed.), Ecological and behavioral methods for the study of bats (pp. 211-220). Washington: Smithsonian Institution Press.

38. Williams-Guillén, K., Olimpi, E., Maas, B., Taylor, P. J., \& Arlettaz, R. (2016). Bats in the Anthropogenic Matrix: Challenges and Opportunities for the Conservation of Chiroptera and Their Ecosystem Services in Agricultural Landscapes. In C. C. Voigt \& 
Kingston, T. (Ed.), Bats in the Anthropocene: Conservation of bats in a changing world (pp. 151 -186). New York: Springer.

39. Wolton, R. J., Arak, P.A., Godfray, H.C.J.,\& Wilson, R.P. (1982). Ecological and behavioural studies of the Megachiroptera at Mont Nimba, Liberia, with notes on Microchiroptera. Mammalia, 46 (4): 419-444.

40. Yamagiwa, J., Kaleme, K., Mwanga, M., \& Basabose, K. (1996). Food density and ranging patterns of Gorillas and Chimpanzees in the Kahuzi-Biega National Park, Zaïre. Tropics, 1/2:

41. 65-77. 